\title{
Yoga lessons for consciousness research: a paralimbic network balancing brain resource allocation
}

\author{
Hans C. Lou ${ }^{1 *}$, Morten Joensson ${ }^{1,2}$ and Morten L. Kringelbach ${ }^{1,2,3 *}$ \\ ${ }^{1}$ Centre for Functionally Integrative Neuroscience, Aarhus University, Aarhus, Denmark \\ ${ }^{2}$ Department of Psychiatry, Warneford Hospital, University of Oxford, Oxford, UK \\ ${ }^{3}$ Department of Psychiatry, Oxford Centre for Human Brain Activity, University of Oxford, Oxford, UK
}

\section{Edited by:}

Jeroen J. A. Van Boxtel, University of California Los Angeles, USA

\section{Reviewed by:}

Zoran Josipovic, New York University, USA

Andrea Eugenio Cavanna, BSMHFT and University of Birmingham, UK

*Correspondence:

Hans C. Lou, Centre for Functionally Integrative Neuroscience, Aarhus

University, Aarhus 8000, Denmark. e-mail: hanslou1@gmail.com;

Morten L. Kringelbach, Department of Psychiatry, Warneford Hospital, University of Oxford, Oxford OX3 7JX, UK.

e-mail:morten.kringelbach@psych. ox.ac.uk
Consciousness has been proposed to play a key role in shaping flexible learning and as such is thought to confer an evolutionary advantage. Attention and awareness are the perhaps most important underlying processes, yet their precise relationship is presently unclear. Both of these processes must, however, serve the evolutionary imperatives of survival and procreation. They are thus intimately bound by reward and emotion to help to prioritize efficient brain resource allocation in order to predict and optimize behavior. Here we show how this process is served by a paralimbic network consisting primarily of regions located on the midline of the human brain. Using many different techniques, experiments have demonstrated that this network is effective and specific for self-awareness and contributes to the sense of unity of consciousness by acting as a common neural path for a wide variety of conscious experiences. Interestingly, hemodynamic activity in the network decreases with focusing on external stimuli, which has led to the idea of a default mode network. This network is one of many networks that wax and vane as resources are allocated to accommodate the different cyclical needs of the organism primarily related to the fundamental pleasures afforded by evolution: food, sex, and conspecifics. Here we hypothesize, however, that the paralimbic network serves a crucial role in balancing and regulating brain resource allocation, and discuss how it can be thought of as a link between current theories of so-called "default mode," "resting state networks," and "global workspace." We show how major developmental disorders of self-awareness and self-control can arise from problems in the paralimbic network as demonstrated here by the example of Asperger syndrome. We conclude that attention, awareness, and emotion are integrated by a paralimbic network that helps to efficiently allocate brain resources to optimize behavior and help survival.

Keywords: consciousness, attention, reward, pleasure, cingulate cortex, orbitofrontal cortex, precuneus, paralimbic network

\section{INTRODUCTION}

Biological evolution is a complex process allowing species and organisms to try to resist the second law of thermodynamics. Over time, this has led to the evolution of increasingly sophisticated brains allowing organisms to adapt and survive longer by learning from experience and predicting future events. Yet, this increase in adaptability also leads to an increasingly difficult resource allocation problem of how to best select between competing wants and likes (Kringelbach and Berridge, 2009). Human consciousness can be thought of as one particular successful solution to this taxing problem, primarily facilitated by the processes of attention and awareness.

The scientific study of human consciousness is growing beyond its infancy and many interesting properties of its underlying neuronal substrates have been established (Changeux and Lou, 2011). Yet countless fundamental discoveries remain, however, and in particular understanding the precise relationship between attention and awareness has remained elusive (although this special issue is beginning to address the issue). In addition to the main tenet of the present discussion, namely of a bidirectional and intimate relationship between attention and self-awareness networks, it should be noted that in particular for the attentional network, there are asymmetries between hemispheric involvement. For instance, Mesulam (1999) drew attention to the prime role of the right hemisphere in generating spatial neglect, and more recently it has been demonstrated that the functional connectivity of the insula to the paralimbic network is asymmetrical as well (Cauda et al., 2011).

One way to conceptualize the relationship between attention and awareness is to think of attention as an executive function allowing the focusing of the mind, while awareness is its closely associated counterpart at the highest level of sensory processing. Yet, this conceptualization leaves out the importance of reward and emotion in guiding the crucial decision making involved in optimizing resource allocation of brain resources, which we discuss in this review. 
Some progress has been made in identifying how self-awareness can help in shaping the decision making. Interestingly, part of this progress has been made possible by studying the introspective states involved in meditation. Studies of highly experienced Yoganidra practitioners have shown that there are significantly different brain networks involved in the states related to relaxation meditation (Yoga-nidra) and normal resting state (i.e., at rest, without intended motor, or mental function), and that these networks vary greatly with the changing contents of consciousness during the two conditions (Lou et al., 1999). In contrast, common to both states and traits is sustained activity in a paralimbic network consisting of midline frontal regions (anterior cingulate and medial prefrontal cortices), parietal regions (primarily precuneus), and striatum (Kjaer and Lou, 2000; see Figures 1 and 2).

This discovery led to the hypothesis that the paralimbic network contributes to the regulation of conscious experience by acting as a common reference of self-perspective for changing conscious states and traits (Kjaer and Lou, 2000). Such a role for the paralimbic network would be consistent with ontogenetic considerations of brain development (Feinberg and Keenan, 2005). Accordingly, animals with a paleomammalian brain, a brain with developed limbic system, but scarcely developed neocortex, may display quite sophisticated social behaviors like maternal nurturing, separation distress calls, and play, while the adjacent neocortex is essential for perception of the self in space and time, a prerequisite for abstract thinking.

The neuroanatomical evidence also supports the hypothesis that the paralimbic network plays a special role in guiding the

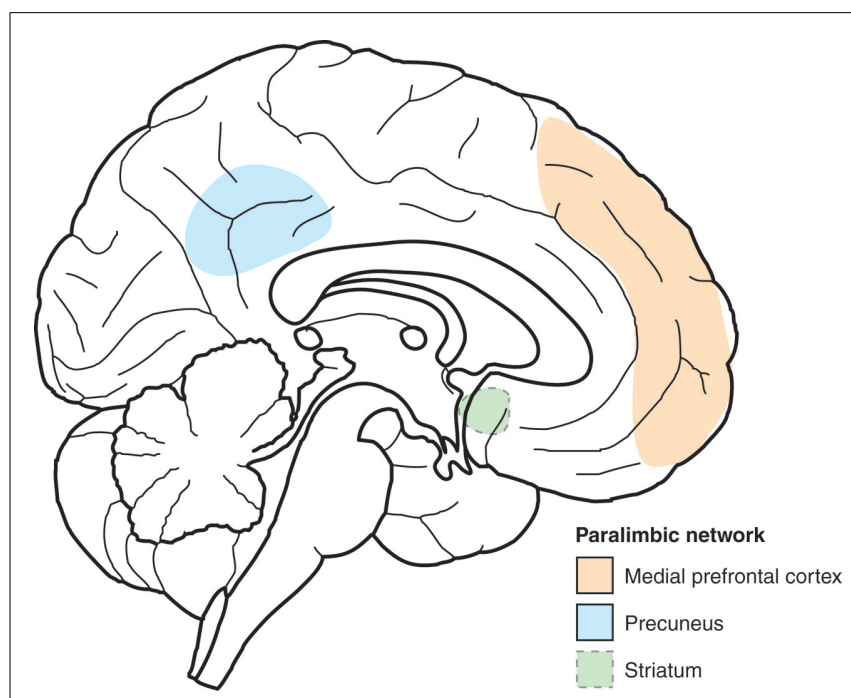

FIGURE 1 | Sketch of the paralimbic network. Principal component analysis was used to identify brain regions common to conscious experiences during yoga-nidra meditation and the normal resting conscious state. Two major clusters were found, explaining 25 and $18 \%$ of the variability. The paralimbic network consists of a set of three regions which contributed to both clusters: Striatum, medial prefrontal, and medial parietal cortices (precuneus). Being active in a wide variety of conscious experiences, we hypothesize that this paralimbic network represented self-reference, as a common denominator for conscious experiences to account for a sense of unity of consciousness. allocation of brain resources by mediating between attention, awareness, and emotion. The paralimbic regions are anatomically located at the interface between the limbic and the neocortical brain, the former providing information on the bodily state and emotions, while the heteromodal neocortical association regions provide extrapersonal sensory and mnemotic information on the highest level of integration available for attention and awareness. Relying on this broad outline and on anatomical, physiological, and behavioral experiments in the monkey, Mesulam (1998) proposed that the cerebral cortex is organized into five hierarchically arranged subtypes: Limbic, paralimbic, heteromodal association, unimodal association, and primary sensory motor regions.

In the present review we synthesize the evidence from the literature to show that the paralimbic network is active and efficient in self-processing, a prime organizer of conscious experience. We show how the paralimbic network uses information from both the external world and introspection to balance the resource allocation, and how the access and balance in this system is regulated by emotion and by neurotransmitters such as dopamine. Finally, we show how disturbances in the paralimbic network may be involved in developmental disorders such as autism.

Before proceeding, however, a brief note of caution regarding the limits of neuroimaging. As such it is important to realize that neuroimaging findings resulting from a comparison of active and control conditions usually are correlative only and will not imply causality. Also they will not necessarily uncover the full network of brain regions involved. Instead, what neuroimaging may reveal are the changes in activity in the important nodes and hubs of the human brain which can bring about changes in the balance of resource allocation between different brain networks, the associated behavior, and the resulting experience. In addition, also electromagnetic methods may have important caveats. One example is the fact that microsaccades may elicit gamma synchrony in the frontal cortex, while the parietal lobe is

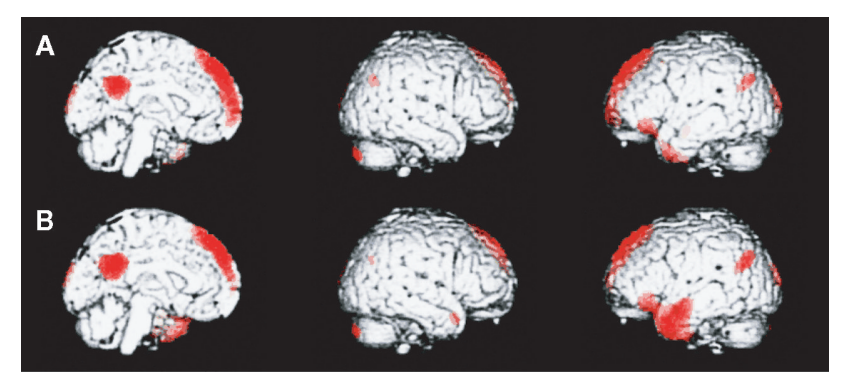

FIGURE 2 | A medial paralimbic network common to conscious experiences. The figure shows brain maps of activity related to self and other (midline, right, and left hemisphere). (A) Emergence of self-representation. Differential activity is noted in medial paralimbic regions, together with bilateral occipital and parietal regions $(p<0.05$, corrected for multiple comparisons). (B) Emergence of representation of other (Danish Queen). Differential activity is noted in medial paralimbic regions, together with bilateral occipital and parietal regions and a confluent left inferior prefrontal and temporal region. Activity in the paralimbic network is similar across the two conditions, an indication of the pivotal role of this network in higher-order consciousness with self-reference. (Lou et al., 2004, with permission). 
unaffected (Carl et al., 2012). The parietal lobe is a key constituent of the paralimbic network discussed in this review. Therefore the gamma synchrony described here cannot be attributed to eye-movements.

\section{THE PARALIMBIC NETWORK IS EFFECTIVE AND SPECIFIC IN SELF-PROCESSING}

The conscious sense of self serves as a common reference for making decisions about the allocation of resources ensuring survival, whether related to any of the fundamental pleasures of food, sex, or other people or even higher-order pleasures such as music or monetary reward. Establishing the functional neuroanatomy of the self is thus potentially a fruitful strategy for identifying the hubs and key nodes in the brain networks important for maintaining the balance between the competing processes of attention, awareness, and emotion.

One essential role for the self is to be a common reference point in space and time for conscious experiences (Gallagher, 2000). Together, they are all experienced in unity, coherent in space and time. Even when someone enters the room, or the phone rings, and the scene and our focus of attention shifts completely, we usually experience the changed scene as continuous with the preceding (Tononi and Edelman, 1998). To account for this coherence of widely different conscious percepts, the self offers a possibility: per definition, we cannot have free-floating sensations with no self to experience them, and we cannot have a self completely devoid of sensory experiences, memories, or feelings. In other words: nonself sensations and memories and the self are two sides of the same coin.

If each of the different, ever-changing sensations and memories are attached to the same coherent self-structure across space and time, these types of information will be bound to each other via the self. A coherent self across time requires a system to retrieve memories of personal experiences, i.e., episodic memory. Correlational evidence suggests that this system is involved not only in retrieval of past personal memories, but also in conceptualizing the future (Ingvar, 1985; Andreasen et al., 1995). Consequently, episodic memory retrieval appears as an indispensable component of the more complex forms of self-awareness and consciousness, which are described in various terms such as extended self, meta-consciousness, autonoetic consciousness, or narrative self (Gallagher, 2000). In a particular amnesic syndrome, transient global amnesia, a rudimentary sense of self is preserved. This state is characterized by loss of episodic memory, while working memory, and semantic memory are relatively spared (Quinette et al., 2003).

The neural organization of episodic memory retrieval involves hemodynamically synchronized activity in medial paralimbic regions including the anterior cingulate and posterior cingulate cortices and adjacent neocortical structures, as well as in the thalamus (Henson et al., 1999; McDermott et al., 1999; Wiggs et al., 1999; Cabeza and Nyberg, 2000; Gardiner, 2001; Tulving, 2002). Further hemodynamic studies have confirmed that the paralimbic network is active in self-awareness (Kjaer et al., 2002b), and single pulse transcranial magnetic stimulation confirmed the specificity and causality of the network in extended self-awareness (Lou et al., 2004; see Figure 3).

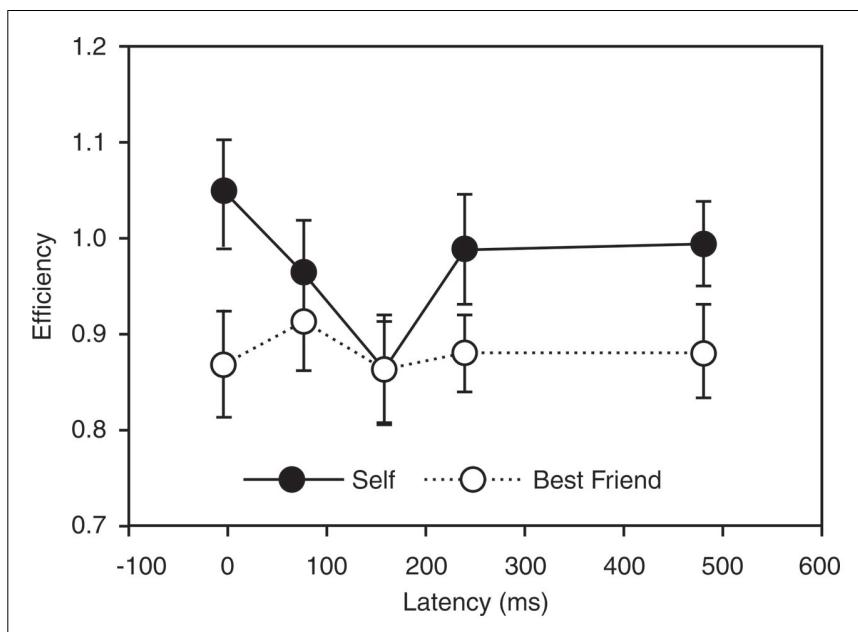

FIGURE 3 | Causality and specificity of one node of the paralimbic network in self-reference. The figure demonstrates the effects of applying transcranial magnetic stimulation (TMS) in targeting the

precuneus/posterior cingulate region in a task probing self-reference. The causality of this region in self-reference is seen by the fact that retrieval of self-judgment was significantly less efficient with TMS at a latency of $160 \mathrm{~ms}$ than with a latency of $0 \mathrm{~ms}(p=0.003)$, suggesting that neural activity at that time interval after stimulus presentation is particularly important for self-representation. Specificity of this region in self-reference is provided by the fact that the difference between self and other (best friend) is significant at most latencies ( $p<0.05)$, except at the specific causal latency. (Lou et al., 2004, with permission).

In contrast to extended self-awareness, the minimal self is prereflexive, immediate, normally infallible, and involves the sense of ownership of experiences. For the minimal self, data on the neural organization are scarcer, but indicate that the same paralimbic structures are active here as well (Vogeley et al., 2004), being a "common denominator" for the self, independent of its complexity, and consisting mainly of a network of paralimbic regions. Functional magnetic resonance imaging (fMRI) have shown a consistent pattern of activity in self-processing for a paralimbic network including anterior cingulate/medial prefrontal cortices, posterior cingulate/medial parietal cortices (with precuneus), and pulvinar thalamus (Lou et al., 2004). In addition, other brain regions including striatum and cerebellum have also been implicated.

This network of paralimbic regions not only shows correlated activity with self-reference, but has also been shown to be causally related to self-reference. Transcranial magnetic pulses (TMS) can transiently disturb the neural function of key regions in the paralimbic network with decreased efficiency specifically of self-reference as a result (Lou et al., 2004).

Anatomically, the medial prefrontal/anterior cingulate and medial parietal/posterior cingulate regions are connected directly via the cingulum bundle, and indirectly, through their rich connections via the "limbic" and intralaminar thalamic nuclei, located centrally at the base of the forebrain (Lou et al., 2004).

Each structure in the paralimbic circuitry contributes fundamental properties to extended self-awareness (Northoff and Bermpohl, 2004). The evidence shows that many of the regions on 
the midline of the prefrontal cortex are involved in self-reference. The medial orbitofrontal cortex at the base of the frontal lobes has been shown to be a related to evaluating the valence of the convergence of intero- and exteroceptive stimuli, including the remarkable valence of human infants (Nauta, 1971; Kringelbach and Rolls, 2004; Kringelbach et al., 2008). This region has been called the "entrance door to self-awareness" based on EEG and MEG studies showing comparatively early engagement after onset of emotional stimuli (Northoff and Bermpohl, 2004). Self-referential stimuli are then monitored in the dorsal anterior cingulate cortex in the sense that stimuli are selected here among competing stimuli for access to consciousness. The supplementary motor area is closely functionally related to the anterior cingulate cortex in preparation for action, in particular when there are no external cues to tell the subject what to do. Self-referential stimuli are thought to be evaluated in the dorso-medial prefrontal cortex as judged to be pertaining to one-self or other persons (Northoff and Bermpohl, 2004). "Theory of the mind," or attributing mental states to others, is also associated with activity in the region, which therefore may constitute a link between introspection and understanding others (Frith and Frith, 1999).

These frontal monitoring and evaluative functions of selfawareness are complemented by functions in the posterior midline establishing spatial and diachronic unity of self and of consciousness: Spatial organization in a first person framework involves posterior cingulate/medial parietal cortex (Northoff and Bermpohl, 2004) which is also active in linking new information with prior knowledge on the subject matter (Maguire et al., 1999). In autonoetic consciousness, the region is active in retrieval of episodic memory for autobiographical self-consciousness (Cavanna and Trimble, 2006). The functional integrity of medial parietal cortex is critical for extended self-awareness. This has been shown by TMS targeting precuneus to transiently disrupt the normal function of this region (Lou et al., 2004). The central role of the intralaminar nuclei for consciousness is illustrated by the fact that even minute lesions here may cause loss of consciousness (Schiff, 2008).

\section{THE ROLE OF THE PARALIMBIC NETWORK IN BALANCING THE DYNAMICS BETWEEN NETWORKS}

Neuroimaging experiments support the proposed role of the paralimbic network as a central integrator and arbitrator of resource allocation in the human brain. In particular, in addition to evidence from indirect techniques such as fMRI and PET, more direct and faster methods such as magnetoencephalography (MEG) have recently provided very useful information regarding the dynamics of the underlying physiology.

When sampling data from the most important hubs of the paralimbic network including the anterior cingulate/medial prefrontal and posterior cingulate/medial parietal cortices as well as thalamic regions, it has been demonstrated that gamma synchrony is a common neural event in both minimal self-reference and extended self-reference, where the degree of synchrony is clearly related to the degree of self-reference (Lou et al., 2010). As noted above, this mechanism of gamma synchrony is not likely to be affected by the recent discovery that microsaccades may give rise to gamma oscillations. These gamma oscillations are induced by extra cerebral sources primarily in the frontal lobe, and have not been found in the parietal cortices (Carl et al., 2012). In addition we used beamforming for source localization which allows a clear distinction between sources.

The degree of gamma synchrony in the paralimbic network is a potential mechanism between conscious experiences and widely different degrees of self-reference. This mechanism of gamma synchrony in the paralimbic network thus meets one of the main requirements needed to bind conscious experience in the so-called neuronal global workspace theory (Dehaene et al., 1998) based on earlier theoretical work (Baars, 1989).

Yet, theories of consciousness abound and while the global workspace theory has been highly influential, other potential candidate theories exist. An equally important concept developed over the last decade is the default mode theory (Gusnard and Raichle, 2001; Raichle et al., 2001). According to this theory, sensory information arriving from the outside world will lead to a decrease in perfusion and energy metabolism of the medial default mode network, in analogy with the re-direction of brain activity with shifting modalities of sensory stimulation (Lam et al., 1999).

At first glance these two theories would seem contradictory, with the global workspace theory referring to a single global brain network processing consciousness, while the default mode theory posits one brain network for processing the outer world, and another network for the inner world.

The hypothesized paralimbic network offers a solution to this apparent paradox. The evidence from MEG, which is a direct measure of brain activity, shows that paralimbic network becomes synchronized to varying degrees during experiences of both the outer world with only minimal self-reference, and more so in introspection during extended self-reference (Lou et al., 2010).

In contrast, we reanalyzed some of our data and found that the paralimbic network showed a significant reduction in gamma power only during a minimally self-related task (syllable counting, see Figure 4), while there were no significant change in gamma power during extended self-reference (retrieval of own judgment on one-self).

Compare this with the evidence from indirect measures of brain activity such as fMRI which shows that regions involved in the default mode network have reduced blood oxygenation level dependent (BOLD) signal during goal directed (non-selfreferential) tasks relative to rest (Raichle et al., 2001). The reduction in gamma power as measured with MEG during an active task (syllable counting) could reflect the deactivation in the default mode network during active tasks. Some of the evidence suggests that changes in gamma power are indeed correlated with BOLD which is an indirect measure of the synaptic activity (Logothetis and Wandell, 2004).

In sum, the evidence from MEG studies show that the paralimbic network can provide the gamma synchrony needed for the global coherent workspace of consciousness, while also fulfilling the criteria of reduced activity (i.e., changes in gamma power in minimal self-reference) in the midline paralimbic regions of self-reference elicited by minimal self-reference.

Yet, general gamma synchrony on its own is not sufficient for consciousness but would also seem to require amplification of activity within recurrent networks. This is suggested by some 


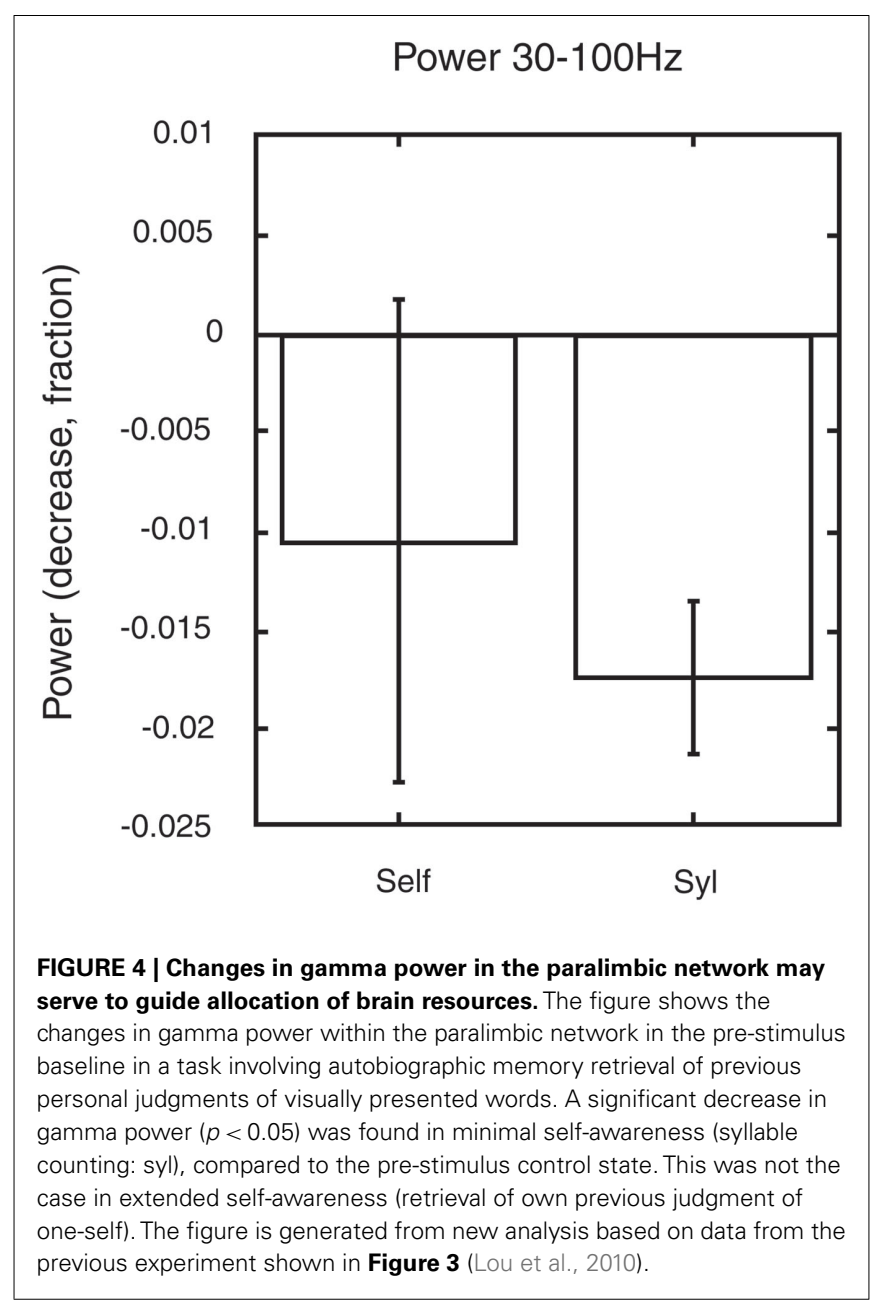

intriguing data gathered over 30 years ago, when (Libet, 1982) discovered that conscious sensations emerge as a function of both intensity and duration of electric stimulation of the human somatosensory cortices. They reported a delay of conscious experience of approximately $400 \mathrm{~ms}$ from onset of a sustained stimulus at threshold intensity and assumed that this latency reflected the need for maintaining the initial frequency and amplitude for an extended period of time. This suggests that amplification of perceptual processing is required to elicit conscious experience, a process which in the neuronal global workspace theory is termed "ignition." In addition, evidence also indicates that high signal intensity in cortico-thalamic interaction is essential for emergence of consciousness. The possible nature of such amplification has been widely discussed in the literature. Several researchers have speculated that extensive recursive activity to bootstrap neuronal processing is a general mechanism for stimuli to become available for consciousness (Tononi and Edelman, 1998).

In spite of this long-standing interest in recurrent neuronal activity, beyond some demonstrations in primary visual cortices, such activity was only recently demonstrated in higherorder, modality non-specific regions using MEG and the so-called Granger causality analysis, a mathematical method initially developed to analyze econometric data but now also used to identify the directionality of flow of information between brain regions (Granger, 1969).

Autobiographic memory retrieval of previous personal judgments of visually presented words was used to probe the temporal flow of activity within the paralimbic network (Lou et al., 2011a). It was demonstrated that the pre-stimulus condition is characterized by causal, recurrent oscillations which are maximal in the lower gamma range. When retrieving previous judgments of visually presented adjectives, this activity is dramatically increased during the stimulus task as ascertained by Granger causality analysis, demonstrating not only recurrent gamma activity in higherorder, modality non-specific regions but also how the paralimbic network serves to allocate brain sources.

\section{CONSCIOUS EXPERIENCE UNDER THE INFLUENCE OF DOPAMINE}

The paralimbic network described here does not only include midline structures in medial anterior and posterior regions which are continuously active in different states and traits of consciousness, but the evidence has also implicated the striatum. It is well-known that dopaminergic neurotransmission is abundantly present in the striatum. Therefore interest has recently focused on the possible role of dopaminergic neurotransmission in the regulation of conscious experience.

At the system level we now know that conscious experience is linked to interacting regions of parietal and prefrontal cortices, which are not only active, but also effective in self-reference (Lou et al., 2004). However, on the molecular level our knowledge has until recently merely been suggestive. Functional brain imaging has established that abnormal conscious experiences in schizophrenia, like hallucinations and delusions, are associated with abnormal dopaminergic neurotransmission (Changeux and Lou, 2011). For instance, striatal dopamine transporter availability is inversely correlated with hallucinations (Schmitt et al., 2006).

Furthermore, clinical evidence indicates that striatal dopamine might regulate conscious experience. One way to measure conscious experience is to approach the problem as one of probability of signal detection (and subsequent subjective interpretation). Following this approach, conscious experience can be determined by the setting of a criterion for when the sensory signal-to-noise ratio warrants sufficient subjective confidence that a stimulus is present. In schizophrenia, psychotic symptoms could be explained as a result of setting too liberal a signal-to-noise criterion. This is thought to be due to abnormally upregulated dopaminergic neurotransmission, an effect of dopamine on the cellular level being to influence the signal-to-noise ratio (Lou et al., 2011b).

Furthermore, in the previously mentioned study of Yoga-nidra meditation it was shown that such meditation is accompanied by a strong increase in sensory awareness, and that this phenomenon is linked to dopamine release in the nucleus accumbens in the ventral striatum, which has been implicated in the paralimbic network consistently active in different conscious states and traits (Kjaer et al., 2002a).

We directly examined the effect of increasing dopamine activation and showed that dopaminergic stimulation with the $\mathrm{D}_{1}$ and $\mathrm{D}_{2}$ receptor agonist pergolide is effective in increasing confidence in seeing words, a valid measure of awareness (Lou et al., 2011b). 
These results demonstrate how dopamine can influence activity across the various nodes and hubs of the paralimbic network, giving rise to changes in both conscious states and content.

\section{THE ROLE OF THE PARALIMBIC NETWORK IN LINKING REWARD, EMOTION, AND SELF-AWARENESS}

The evidence presented above supports the hypothesis that the paralimbic network subserves self-processing in the human brain, and that specific changes in synchrony between the nodes and hubs of this network can regulate the dynamics of widespread connected brain networks. In addition, the evidence also suggests that dopamine has a special role in modulating activity in this network.

The evidence thus supports the overall notion that the paralimbic network plays a key role in the conscious brain resource allocation associated with predicting and selecting behaviors that ensures survival. The fundamental rewards afforded by evolution to ensure survival are food, sex, and conspecifics; and the timecourses of the associated behaviors are cyclical (Kringelbach and Berridge, 2009). There are distinct wanting, liking, and learning phases in the reward cycle, which have been shown to have partly dissociable neural substrates (Kringelbach, 2005).

Over time the human brain has to use attentional mechanisms to detect potential rewards which, depending on the current state of the organism, may (or may not) initiate a specific reward cycle. For example, after becoming aware of hunger signals, the food reward cycle is initiated and our attention invariably turns to finding potential food rewards.

We propose that the paralimbic network plays a key role in integrating awareness, attention, and emotion processing to optimize the brain resource allocation. The inputs from the senses such as vision, auditory, taste, smell, and touch (linked to survival-related rewards such as food and sex) and their subsequent evaluation have the potential to temporarily shift the focus of the brain networks to allow for efficient processing and control over behaviors (Berridge and Kringelbach, 2008; Kringelbach et al., 2008). The paralimbic network helps to ensure that the processing remains balanced over longer time periods.
Mounting evidence has demonstrated that frontal regions such as the medial orbitofrontal and the anterior cingulate cortices are situated anatomically at the crossroad between interacting networks of attention, awareness, and emotion as necessary nodes linking the networks (Tsuchiya and Adolphs, 2007; Kringelbach et al., 2011). It should, however, be noted that these regions are heterogenous regions with many sub-regions which have been implicated in different networks (Kringelbach and Rolls, 2004; Beckmann et al., 2009).

Direct causal evidence for the interaction has been given by studies showing that direct electrical stimulation of the anterior cingulate cortex can help to alleviate severe chronic pain in patients (Kringelbach et al., 2010). Recently, we further demonstrated that reward can directly impact conscious experience and this integration is directly reflected in the neural activity in the anterior cingulate cortex as measured by local field potentials (Rømer Thomsen et al., 2011).

In general, emerging evidence shows that perturbations to the paralimbic network may manifest in an unbalancing of widespread brain network as seen for example in the anhedonia associated with mental illness (Kringelbach et al., 2011).

\section{CLINICAL CONSEQUENCES OF DEVELOPMENT IMPEDIMENT OF THE PARALIMBIC NETWORK}

Development perturbations to the paralimbic network are not uncommon (Lou, 2011). Here we will briefly focus on an example of developmental dysfunction found in the Asperger syndrome (see Box 1).

Individuals with Asperger syndrome would appear to have problems with introspection and narrative self-awareness. In particular they have difficulty identifying and reflecting on their own emotional states. They also have peculiar concrete thought patterns and a tendency to focus on external events rather than inner experiences (Hill et al., 2004).

Given the hypothesis of the paralimbic network presented here, this failure of subjectivity may be predicted to be linked to dysfunction in the paralimbic loop and medial neocortical structures. While still scarce, some of the experimental data would seem to support this link. One neuroimaging study involved higher-order

\section{Box 1 | Asperger syndrome.}

Autism is a neurodevelopmental disorder of genetic origin, characterized by impaired social interaction and communication as well as repetitive behavior and restricted interests. The symptomatology being highly variable, the condition is often referred to as "autism spectrum disorder." Asperger syndrome is a subgroup where the affected individuals have normal language development and normal or above normal $I Q$, together with the typical social communication impairments, obsessions, and narrow interests, suggesting impaired binding of attention, awareness, and emotion.

Peter is a young boy 16 years of age. After a normal pregnancy and birth he was delayed in motor development and slightly delayed in development of language. He did not babble until he suddenly spoke perfectly correct. As a toddler he was always playing with construction toys and puzzles, but not with other children. At school he is marginalized, having special interests not shared by his classmates. He behaves oddly in social interaction where he is rigid and clumsy, with a very formal, monotonous language. He is unable to interpret moods or motives of others. He speaks without restraint about his special interests, in an exalted, gesturing, and noisy way. The psychological investigation shows that he is of normal intelligence, with high scores in memory of facts, especially meaningless facts, and he is eminent at puzzles and labyrinths. In contrast, he scores below normal in reasoning and social comprehension, in particular sequential understanding of social activities. He is unable to generate inclusive terms covering a variety of objects. He is characterized by orienting to details. He is diagnosed as having Asperger syndrome, a milder variant of autism. 
consciousness based on linguistic information, sentence comprehension (Just et al., 2004). In autists compared to normal controls, higher activity was seen in the Wernicke region, while lower activity was found in Broca's region and in the medial paralimbic regions. This finding supports the hypothesis that the medial paralimbic circuitry could be defective in autism. Yet, some caution is needed given that the autists and the normal control participants were not compared directly, but only indirectly via a common reference to a fixation point.

Deficiency of the paralimbic loop of self-reference could potentially be explained by the evidence for volume reduction and reduction in glucose metabolism in the entire cingulate cortex in autism (Haznedar et al., 2000, 2004). In fact, related deficiencies may be seen in another disorder of self-awareness and higher-order consciousness: schizophrenia (Haznedar et al., 2004).

\section{CONCLUSION AND PERSPECTIVE}

There is a clear lesson for consciousness research initially emerging from studying the brain activity in experienced Yoga-nidra teachers during meditation but subsequently supported by a

\section{REFERENCES}

Andreasen, N. C., O'Leary, D. S., Cizadlo, T., Arndt, S., Rezai, K., Watkins, G. L., Ponto, L. L., and Hichwa, R. D. (1995). Remembering the past: two facets of episodic memory explored with positron emission tomography. Am. J. Psychiatry 152, 1576-1585.

Baars, B. J. (1989). A Cognitive Theory of Consciousness. Cambridge, MA: Cambridge University Press.

Beckmann, M., Johansen-Berg, H., and Rushworth, M. F. (2009). Connectivity-based parcellation of human cingulate cortex and its relation to functional specialization. $J$. Neurosci. 29, 1175-1190.

Berridge, K. C., and Kringelbach, M. L. (2008). Affective neuroscience of pleasure: reward in humans and animals. Psychopharmacology (Berl.) 199, 457-480.

Cabeza, R., and Nyberg, L. (2000). Imaging cognition II: an empirical review of 275 PET and fMRI studies. J. Cogn. Neurosci. 12, 1-47.

Carl, C., Acik, A., Konig, P., Engel, A. K., and Hipp, J. F. (2012). The saccadic spike artifact in MEG. Neuroimage 59, 1657-1667.

Cauda, F., D’Agata, F., Sacco, K., Duca, S., Geminiani, G., and Vercelli, A. (2011). Functional connectivity of the insula in the resting brain. $\mathrm{Neu}$ roimage $55,8-23$.

Cavanna, A. E., and Trimble, M. R. (2006). The precuneus: a review of its functional anatomy and behavioural correlates. Brain 129, 564-583.

Changeux, J. P., and Lou, H. C. (2011). Emergent pharmacology of conscious experience: new perspectives in substance addiction. FASEB J. 25, 2098-2108.

Dehaene, S., Kerszberg, M., and Changeux, J. P. (1998). A neuronal model of a global workspace in effortful cognitive tasks. Proc. Natl. Acad. Sci. U.S.A. 95, 14529-14534.

Feinberg, T. E., and Keenan, J. P. (2005). The Lost Self: Pathologies of the Brain and Identity. Oxford: Oxford University Press.

Frith, C. D., and Frith, U. (1999). Interacting minds - a biological basis. Science 286, 1692-1695.

Gallagher, I. I. (2000). Philosophical conceptions of the self: implications for cognitive science. Trends Cogn. Sci. (Regul. Ed.) 4, 14-21.

Gardiner, J. M. (2001). Episodic memory and autonoetic consciousness: a first-person approach. Philos. Trans. R. Soc. Lond. B Biol. Sci. 356, 1351-1361.

Granger, C. W. J. (1969). Investigating causal relations by econometric models and cross-spectral methods. Econometrica 37, 424-438.

Gusnard, D. A., and Raichle, M. E. (2001). Searching for a baseline: functional imaging and the resting human brain. Nat. Rev. Neurosci. 2, 685-694.

Haznedar, M. M., Buchsbaum, M. S., Hazlett, E. A., Shihabuddin, L., New, A., and Siever, L. J. (2004). Cingulate gyrus volume and metabolism in the schizophrenia spectrum. Schizophr. Res. 71, 249-262.

Haznedar, M. M., Buchsbaum, M. S., Wei, T. C., Hof, P. R., Cartwright, C., Bienstock, C. A., and Hollander, E. (2000). Limbic circuitry in

wealth of other experiments: a common paralimbic network serves to regulate and balance the dynamic resource allocation needed to ensure survival. This process is guided by processes linked to awareness, attention, and emotion in order to support memorydependent self-reference, which in narrative self-consciousness is extended into adjacent neocortical regions. Dire clinical consequences are linked to its impediment in pathological conditions. Yet, as a common reference of conscious experiences, it provides us with an adaptive tool for building a unified self and personality over the years by successfully balancing the allocation of brain resources for survival and procreation.

\section{ACKNOWLEDGMENTS}

The authors are grateful to Professor Per Hove Thomsen (Department of Child Psychiatry, Aarhus University Hospital, Aarhus, Denmark) for the case story on Asperger syndrome, as well as the Mind Lab supported by the Danish Ministry for Science, Technology and Innovation, The Lundbeck Foundation and TrygFonden Charitable Foundation for financial support for some of the research discussed.

patients with autism spectrum disorders studied with positron emission tomography and magnetic resonance imaging. Am. J. Psychiatry 157, 1994-2001.

Henson, R. N., Rugg, M. D., Shallice, T., Josephs, O., and Dolan, R. J. (1999). Recollection and familiarity in recognition memory: an eventrelated functional magnetic resonance imaging study. J. Neurosci. 19 , 3962-3972.

Hill, E., Berthoz, S., and Frith, U. (2004) Brief report: cognitive processing of own emotions in individuals with autistic spectrum disorder and in their relatives. J. Autism Dev. Disord. 34, 229-235.

Ingvar, D. H. (1985). "Memory of the future": an essay on the temporal organization of conscious awareness. Hum. Neurobiol. 4, 127-136.

Just, M. A., Cherkassky, V. L., Keller, T. A., and Minshew, N. J. (2004). Cortical activation and synchronization during sentence comprehension in high-functioning autism: evidence of underconnectivity. Brain 127, 1811-1821.

Kjaer, T. W., Bertelsen, C., Piccini, P., Brooks, D., Alving, J., and Lou, H. C. (2002a). Increased dopamine tone during meditation-induced change of consciousness. Brain Res. Cogn. Brain Res. 13, 255-259.

Kjaer, T. W., Nowak, M., and Lou, H. C. (2002b). Reflective selfawareness and conscious states: PET evidence for a common midline parietofrontal core. Neuroimage 17, 1080-1086.

Kjaer, T. W., and Lou, H. C. (2000). Interaction between precuneus and dorsolateral prefrontal cortex may play a unitary role in consciousness: a principal component analysis of rCBF. Conscious. Cogn. 9, S59.

Kringelbach, M. L. (2005). The orbitofrontal cortex: linking reward to hedonic experience. Nat. Rev. Neurosci. 6, 691-702.

Kringelbach, M. L., and Berridge, K. C. (2009). Towards a functional neuroanatomy of pleasure and happiness. Trends Cogn. Sci. (Regul. Ed.) 13, 479-487.

Kringelbach, M. L., Green, A. L., and Aziz, T. Z. (2011). Balancing the brain: resting state networks and deep brain stimulation. Front. Integr. Neurosci. 5:8. doi:10.3389/fnint.2011.00008

Kringelbach, M. L., Green, A. L., Owen, S. L., Schweder, P. M., and Aziz, T. Z. (2010). Sing the mind electric-principles of deep brain stimulation. Eur. J. Neurosci. 32, 1070-1079.

Kringelbach, M. L., Lehtonen, A., Squire, S., Harvey, A. G., Craske, M. G., Holliday, I. E., Green, A. L., Aziz, T. Z., Hansen, P. C., Cornelissen, P. L., and Stein, A. (2008). A specific and rapid neural signature for parental instinct. PLoS ONE 3, e1664. doi:10.1371/journal.pone.0001664

Kringelbach, M. L., and Rolls, E. T. (2004). The functional neuroanatomy of the human orbitofrontal cortex: evidence from neuroimaging and neuropsychology. Prog. Neurobiol. 72, 341-372.

Lam, K., Kakigi, R., Kaneoke, Y., Naka, D., Maeda, K., and Suzuki, H. (1999). Effects of visual and 
auditory stimulation on somatosensory evoked magnetic fields. Clin. Neurophysiol. 110, 295-304.

Libet, B. (1982). Brain stimulation in the study of neuronal functions for conscious sensory experiences. Hum. Neurobiol. 1, 235-242.

Logothetis, N. K., and Wandell, B. A. (2004). Interpreting the BOLD signal. Annu. Rev. Physiol. 66, 735-769.

Lou, H. C. (2011). Paradigm shift in consciousness research: the child's self-awareness and abnormalities in autism, ADHD, and schizophrenia. Acta Paediatr. doi: 10.1111/j.16512227.2011.02456.x. [Epub ahead of print].

Lou, H. C., Gross, J., Biermann-Ruben, K., Kjaer, T. W., and Schnitzler, A. (2010). Coherence in consciousness: paralimbic gamma synchrony of self-reference links conscious experiences. Hum. Brain Mapp. 31, 185-192.

Lou, H. C., Joensson, M., BiermannRuben, K., Schnitzler, A., Ostergaard, L., Kjaer, T. W., and Gross, J. (2011a). Recurrent activity in higher order, modality non-specific brain regions: a Granger causality analysis of autobiographic memory retrieval. PLoS ONE 6, e22286. doi:10.1371/journal.pone.0022286

Lou, H. C., Skewes, J. C., Thomsen, K. R., Overgaard, M., Lau, H. C., Mouridsen, K., and Roepstorff, A. (2011b). Dopaminergic stimulation enhances confidence and accuracy in seeing rapidly presented words. J. Vis. 11, $1-6$.

Lou, H. C., Kjaer, T. W., Friberg, L., Wildschiodtz, G., Holm, S., and
Nowak, M. (1999). A 15O-H2O PET study of meditation and the resting state of normal consciousness. Hum. Brain Mapp. 7, 98-105.

Lou, H. C., Luber, B., Crupain, M., Keenan, J. P., Nowak, M., Kjaer, T. W., Sackeim, H. A., and Lisanby, S. H. (2004). Parietal cortex and representation of the mental Self. Proc. Natl. Acad. Sci. U.S.A. 101, 6827-6832.

Maguire, E. A., Frith, C. D., and Morris, R. G. (1999). The functional neuroanatomy of comprehension and memory: the importance of prior knowledge. Brain 122(Pt 10), 1839-1850.

McDermott, K. B., Ojemann, J. G., Petersen, S. E., Ollinger, J. M., Snyder, A. Z., Akbudak, E., Conturo, T. E., and Raichle, M. E. (1999). Direct comparison of episodic encoding and retrieval of words: an eventrelated fMRI study. Memory 7, 661-678.

Mesulam, M. M. (1998). From sensation to cognition. Brain 121, 1013-1052.

Mesulam, M. M. (1999). Spatial attention and neglect: parietal, frontal and cingulate contributions to the mental representation and attentional targeting of salient extrapersonal events. Philos. Trans. R. Soc. Lond. B Biol. Sci. 354, 1325-1346.

Nauta, W. J. (1971). The problem of the frontal lobe: a reinterpretation. J. Psychiatr. Res. 8, 167-187.

Northoff, G., and Bermpohl, F. (2004). Cortical midline structures and the self. Trends Cogn. Sci. (Regul. Ed.) 8, 102-107.

Quinette, P., Guillery, B., Desgranges, B., de la Sayette, V., Viader, F., and
Eustache, F. (2003). Working memory and executive functions in transient global amnesia. Brain 126, 1917-1934.

Raichle, M. E., MacLeod, A. M., Snyder, A. Z., Powers, W. J., Gusnard, D. A., and Shulman, G. L. (2001). A default mode of brain function. Proc. Natl. Acad. Sci. U.S.A. 98, 676-682.

Rømer Thomsen, K., Lou, H. C., Joensson, M., Hyam, J. A., Holland, P., Parsons, C. E., Young, K. S., Møller, A. Stein, A., Green, A. L., Kringelbach, M. L., and Aziz, T. Z. (2011). Impact of emotion on consciousness: positive stimuli enhance conscious reportability. PLoS ONE 6, e18686. doi:10.1371/journal.pone.0018686

Schiff, N. D. (2008). Central thalamic contributions to arousal regulation and neurological disorders of consciousness. Ann. N. Y. Acad. Sci.1129, 105-118.

Schmitt, G. J., Frodl, T., Dresel, S., la Fougere, C., Bottlender, R., Koutsouleris, N., Hahn, K., Moeller, H. J., and Meisenzahl, E. M. (2006). Striatal dopamine transporter availability is associated with the productive psychotic state in first episode, drug-naïve schizophrenic patients. Eur. Arch. Psychiatry Clin. Neurosci. 256, 115-121.

Tononi, G., and Edelman, G. M. (1998). Consciousness and complexity. Science 282, 1846-1851.

Tsuchiya, N., and Adolphs, R. (2007). Emotion and consciousness. Trends Cogn. Sci. (Regul. Ed.) 11, 158-167.

Tulving, E. (2002). Episodic memory: from mind to brain. Annu. Rev. Psychol. 53, 1-25.
Vogeley, K., May, M., Ritzl, A. Falkai, P., Zilles, K., and Fink, G. R. (2004). Neural correlates of first-person perspective as one constituent of human selfconsciousness. J. Cogn. Neurosci. 16, 817-827.

Wiggs, C. L., Weisberg, J., and Martin, A. (1999). Neural correlates of semantic and episodic memory retrieval. Neuropsychologia 37, 103-118.

Conflict of Interest Statement: The authors declare that the research was conducted in the absence of any commercial or financial relationships that could be construed as a potential conflict of interest.

Received: 04 October 2011; accepted: 21 November 2011; published online: 16 December 2011.

Citation: Lou HC, Joensson $M$ and Kringelbach ML (2011) Yoga lessons for consciousness research: a paralimbic network balancing brain resource allocation. Front. Psychology 2:366. doi: 10.3389/fpsyg.2011.00366

This article was submitted to Frontiers in Consciousness Research, a specialty of Frontiers in Psychology.

Copyright (c) 2011 Lou, Joensson and Kringelbach. This is an open-access article distributed under the terms of the Creative Commons Attribution Non Commercial License, which permits non-commercial use, distribution, and reproduction in other forums, provided the original authors and source are credited. 